\title{
Pathological stage, surgical margin and lymphovascular invasion as prognostic factors after salvage radiotherapy for post-prostatectomy relapsed prostate cancer - outcomes and optimization strategies
}

\author{
Isabel Rodrigues $\mathbb{1}^{1}$, Carolina Ferreira ${ }^{1}{ }^{1,2}$, Joana Gonçalves ${ }^{1,2}$, Luísa Carvalho ${ }^{1}$, Jorge Oliveira ${ }^{2}$, \\ Carla Castro ${ }^{1,2}$, Ângelo Oliveira ${ }^{1,2}$ \\ ${ }^{1}$ External Radiotherapy Department, Instituto Português de Oncologia do Porto Francisco Gentil, E.P.E., Portugal \\ ${ }^{2}$ Urology Clinic, Instituto Português de Oncologia do Porto Francisco Gentil, E.P.E, Portugal
}

\begin{abstract}
Background: Salvage radiotherapy (sRT) is the main potentially curative treatment after biochemical failure/locoregional relapse post-radical prostatectomy (RP). The aim of the study was to characterize the population who underwent sRT after RP at our Department, to understand the influence of several potential prognosis factors, and to determine possible optimization strategies.

Materials and methods: We retrospectively analyzed patients undergoing sRT at our department between 2012 and 2017, evaluating patient, tumor and treatment characteristics, restaging procedures and clinical outcomes - namely biochemical relapse-free survival (BC-RFS), clinical relapse-free survival (C-RFS), additional hormone therapy-free survival (HT-FS) and overall survival (OS). We assessed potential prognostic factors by univariate and multivariate models (MVA).

Results: We included 277 patients (median age 68 years). Median pre-sRT PSA was $>0.5 \mathrm{ng} / \mathrm{mL}$ in $54.9 \%$. All underwent prostate bed irradiation. Pelvic lymph nodes were included in 9.7\%. Outcome analysis was performed for 264 patients (35.6 months median follow-up). At 3 years, BC-RFS was $61.4 \%$, C-RFS was $81.3 \%$, HT-FS was $79.9 \%$ and OS was $96.6 \%$. Most relapses occurred in regional lymph nodes only (47.9\% patients who relapsed). On MVA, lymphovascular invasion, advanced pT-stages and negative margins negatively influenced BC-RFS $(p=0.029, p=0.002$ and $p<0.001)$ and HT-FS $(p=0.001, p=0.029$ and $p=0.002)$. C-RFS was worsened by lymphovascular invasion $(p=0.009)$ and negative margins $(p=0.015)$. These had no effect on OS. BC-RFS and HT-FS were improved when sRT started while PSA $\leq 0.5 \mathrm{ng} / \mathrm{mL}(p<0.05)$.

Conclusion: Lymphovascular invasion, higher pT-stages and negative margins negatively affected prognosis. An early start of sRT (PSA $\leq 0.5 \mathrm{ng} / \mathrm{mL}$ ) predicted better BC-RFS and HT-FS.
\end{abstract}

Key words: prostatic neoplasms; recurrence; radiation therapy; salvage therapy; treatment outcome Rep Pract Oncol Radiother 2021;26(4):535-544

Address for correspondence: Isabel Rodrigues, Serviço de Radioterapia Externa do IPO Porto, Rua Dr António Bernardino de Almeida, 4200-072 Porto, Portugal; e-mail: isabel.rodrigues@ipoporto.min-saude.pt; isabelbastosrodrigues@gmail.com 


\section{Introduction}

Salvage radiotherapy (sRT) is a potentially curative treatment for patients with prostatic bed and/or nodal relapse without distant metastasis after radical prostatectomy (RP). Several factors have been associated with a worse prognosis in this setting, both related to initial disease characteristics and to the relapse itself (such as PSA). Understanding the influence of these factors may contribute to an improvement in patient selection, restaging procedures, sRT timing, sRT technical factors, and surveillance of this particular cohort $[1,2]$. Also, recent prospective studies, namely RADICALS-RT, RAVES and GETUG-AFU 17 [3-5] suggest that adjuvant radiotherapy may not be advantageous for most patients when compared to an early salvage strategy. Hence, identifying prognosis factors may aid in selecting patients for whom this approach can be confidently proposed.

Our purpose was, therefore, to characterize the population who had undergone sRT for relapse after radical prostatectomy (RP) at our Department, to report clinical outcomes, patterns of relapse and prognostic factors, and to determine potential optimization strategies. We also aimed to understand the impact of an early start of sRT after relapse.

\section{Materials and methods}

We performed a retrospective analysis of all patients who had received salvage radiotherapy for biochemical/clinical relapse after RP for prostate cancer at our Department. Information was obtained from clinical records regarding initial disease characteristics and primary treatment, restaging after biochemical relapse (including PSA and imaging studies), treatment after relapse (radiotherapy and hormone therapy) and follow-up (namely radiotherapy side effects, total PSA measurements, imaging studies, further treatments received). We included all patients who suffered a biochemical relapse after RP for prostate cancer (defined by a serum PSA $\geq 0.2 \mathrm{ng} / \mathrm{mL}$ followed by a second confirmatory level, as per ASTRO/AUA and EAU criteria [6]). After performing the restaging procedures (MRI, PET/CT and/or bone scintigraphy, which are further explored in the Results section) and being discussed at a multidisciplinary tumor board, the patients were referenced to our Department for
sRT and treated between $1^{\text {st }}$ January 2012 and $31^{\text {st }}$ December 2017. Since the sRT treatment characteristics were not uniform for all patients (total dose and fractionation, type of boost, treatment volumes, technique), they are analysed in the Results section. We considered the following organs at risk relevant for planning purposes: rectum (V60 <35\% and V50 < 50\%), bladder (maximum dose $<65 \mathrm{~Gy}$, V65 $<50 \%$ ), femoral heads (V52 $<100 \%$ ) and penile-bulb (not dose-limiting, to a medium dose $<50$ Gy or D90 < 50 Gy). Patients were initially staged according to the AJCC 7 th edition. Early sRT was defined as sRT starting with a PSA $\leq 0.5 \mathrm{ng} / \mathrm{mL}$.

\section{Follow-up and toxicity evaluation}

After sRT, patients remained in follow-up, undergoing periodical serum PSA measurements and clinical evaluation for relapse and side effect monitoring, at physician discretion. Biochemical relapse after sRT was defined as a rise in serum PSA $\geq 0.2 \mathrm{ng} / \mathrm{mL}$ above the nadir followed by an equal or higher value [7], or as an absence of serum PSA nadir [8]. For patients under salvage androgen deprivation therapy (ADT), PSA values were included from the moment of ADT suspension. Clinical recurrence was assessed by physical examination, computed tomography (CT), ${ }^{68} \mathrm{Ga}$-PSMA $\mathrm{PET} / \mathrm{CT}$ positron emission tomography/computed tomography (PET/CT), ${ }^{18} \mathrm{~F}$-choline PET/CT and/or bone scintigraphy after biochemical recurrence.

\section{Data collection and analysis}

Data was collected from clinical records, stored and analyzed using IBM SPSS Statistics Version 25. We evaluated the following outcomes: biochemical relapse-free survival (BC-RFS; time from the end of sRT to the date of biochemical relapse), clinical relapse-free survival (C-RFS; time from the end of sRT to the date of clinical relapse, determined by physical examination or imaging studies); additional hormone therapy-free survival (HT-FS; time from end of sRT to the first day of further hormone therapy) and overall survival (OS; time from end of sRT to death). The Kaplan-Meier estimator was used to build survival curves, and survival between groups was compared with the Log-Rank test. Univariate models were built to assess the influence of each disease characteristic on the outcome (including the following: grade group, perineural invasion, lymphovascular invasion, surgical margin, $\mathrm{pT}$ stage, 
initial PSA, presence of undetectable post-RP PSA, and time between RP and relapse). Then, multivariate models retaining significant variables were built to determine the influence of each of them on the prognosis, and hazard ratios were obtained by Cox proportional hazards regression models. The Mann-Whitney test was used to compare PSA values between groups, and the relationship between qualitative variables was determined with the Chi-Square test. For a more simple description of the results, patients were divided into two groups according to pre-sRT PSA: group A $(\leq 0.5 \mathrm{ng} / \mathrm{mL})$ and group $B(>0.5 \mathrm{ng} / \mathrm{mL})$.

\section{Results}

We included 277 patients who were treated at our department from 2012 to 2017, with a median age of 68 years ( 48 to 81 years). Disease and primary treatment characteristics are presented in Table 1.

\section{Primary treatment}

Most patients underwent RP at other hospitals (with only $30.3 \%$ having been primarily treated at our institution). Median pre-RP PSA was 8.2 $\mathrm{ng} / \mathrm{mL}$ and median post-RP PSA was $0.03 \mathrm{ng} / \mathrm{mL}$. Primary tumor was described as Gleason $\leq 7$ for most patients ( $\mathrm{n}=208,75.1 \%)$, and the majority for whom the information was available had perineural invasion $(\mathrm{n}=169,61.0 \%)$ and did not have lymphovascular invasion (reported in $42 \mathrm{pa}-$ tients, $15.2 \%)$. Patients were initially staged as pT2a (8.7\%), pT2b (4.3\%), pT2c (43.3\%), pT3a (30.3\%) or pT3b (11.9\%). Most (75.8\%) did not undergo pelvic lymphadenectomy. Out of the 67 patients who did, a median of 6 lymph nodes were excised, and 4 patients had nodal disease. Positive margins were described for 67 surgical specimens (24.2\%). Most patients did not receive adjuvant hormone therapy ( $\mathrm{n}=257,92.7 \%)$ after radical prostatectomy. Median time from RP to biochemical relapse was 30 months (range: 1-184) and median time from diagnosis of relapse and start of sRT was 9.6 months (range: 0.23-135.7).

\section{Restaging procedures}

Median pre-sRT PSA was 0.59 (IQR 0.77; minimum PSA was 0.137 for patients who were not on ADT). Pre-sRT PSA was $>0.5 \mathrm{ng} / \mathrm{mL}$ for 152 patients (54.9\%). As shown on Figure 1, pelvic
Table 1. Patient and primary disease characteristics

\begin{tabular}{|c|c|c|}
\hline \multicolumn{2}{|l|}{ Characteristic } & Patients \\
\hline Gleason & $\begin{array}{l}6(3+3) \\
7(3+4) \\
7(4+3) \\
8(3+5) \\
8(4+4) \\
9(4+5) \\
9(5+4)\end{array}$ & $\begin{array}{c}28(10.1 \%) \\
96(34.7 \%) \\
84(30.3 \%) \\
7(2.5 \%) \\
42(15.2 \%) \\
18(6.5 \%) \\
1(0.4 \%)\end{array}$ \\
\hline Perineural invasion & $\begin{array}{l}\text { Yes } \\
\text { No } \\
\text { Missing }\end{array}$ & $\begin{array}{c}169(61.0 \%) \\
38(13.7 \%) \\
70(25.3 \%)\end{array}$ \\
\hline Lymphovascular invasion & $\begin{array}{l}\text { Yes } \\
\text { No } \\
\text { Missing }\end{array}$ & $\begin{array}{c}42(15.2 \%) \\
157(56.7 \%) \\
78(28.2 \%)\end{array}$ \\
\hline pT stage & $\begin{array}{l}\text { pT2a } \\
\text { рT2b } \\
\text { pT2c } \\
\text { рT3a } \\
\text { pT3b } \\
\text { Missing }\end{array}$ & $\begin{array}{c}24(8.7 \%) \\
12(4.3 \%) \\
120(43.3 \%) \\
84(30.3 \%) \\
33(11.9 \%) \\
4(1.4 \%)\end{array}$ \\
\hline Surgical margins & $\begin{array}{l}\text { Positive } \\
\text { Negative } \\
\text { Tangential } \\
\text { Missing }\end{array}$ & $\begin{array}{c}67(24.2 \%) \\
165(59.6 \%) \\
31(11.2 \%) \\
14(5.1 \%)\end{array}$ \\
\hline Adjuvant ADT & $\begin{array}{l}\text { Yes } \\
\text { No }\end{array}$ & $\begin{array}{c}20 \text { (7.3\%) } \\
257 \text { (92.7\%) }\end{array}$ \\
\hline Median Pre-RP PSA & $\begin{array}{l}8.2 \mathrm{ng} / \mathrm{mL} \\
\text { (Range: } 2.1\end{array}$ & $\mathrm{ng} / \mathrm{mL}$ ) \\
\hline Median Post-RP PSA & $\begin{array}{l}0.03 \mathrm{ng} / \mathrm{mL} \\
\text { (Range: } 0- \\
\text { Undetectak }\end{array}$ & $\begin{array}{l}\mathrm{g} / \mathrm{mL}) \\
31(29.2 \%)\end{array}$ \\
\hline Median time RP — Relapse & 30 months & \\
\hline Median time relapse $-\mathrm{sRT}$ & 10 months & 36) \\
\hline
\end{tabular}

ADT — androgen deprivation therapy; RP — radical prostatectomy; SRT — salvage radiotherapy

magnetic resonance image (MRI) was requested for 206 patients $(74.4 \%)$ prior to sRT, having identified prostate bed relapse on $44.7 \%$ and additional pelvic nodal relapse on $1.9 \%$. PET/CT imaging was performed on 72 patients: ${ }^{68} \mathrm{Ga}-\mathrm{PSMA} \mathrm{PET} / \mathrm{CT}$ on $51(18.4 \%),{ }^{18} \mathrm{~F}$-choline PET/CT on $22(7.9 \%)$ and both on 1 patient.

Out of the 92 patients whose MRI was negative, $24(26.1 \%)$ underwent a PET study. This technique identified additional prostate bed foci of disease in 4 patients $(16 \% ; 1.8 \%$ of all pts who had an MRI) and nodal disease only in 5 patients $(20 \% ; 2.4 \%$ of all pts who had an MRI). We compared clinical outcomes between the group of patients with nega- 


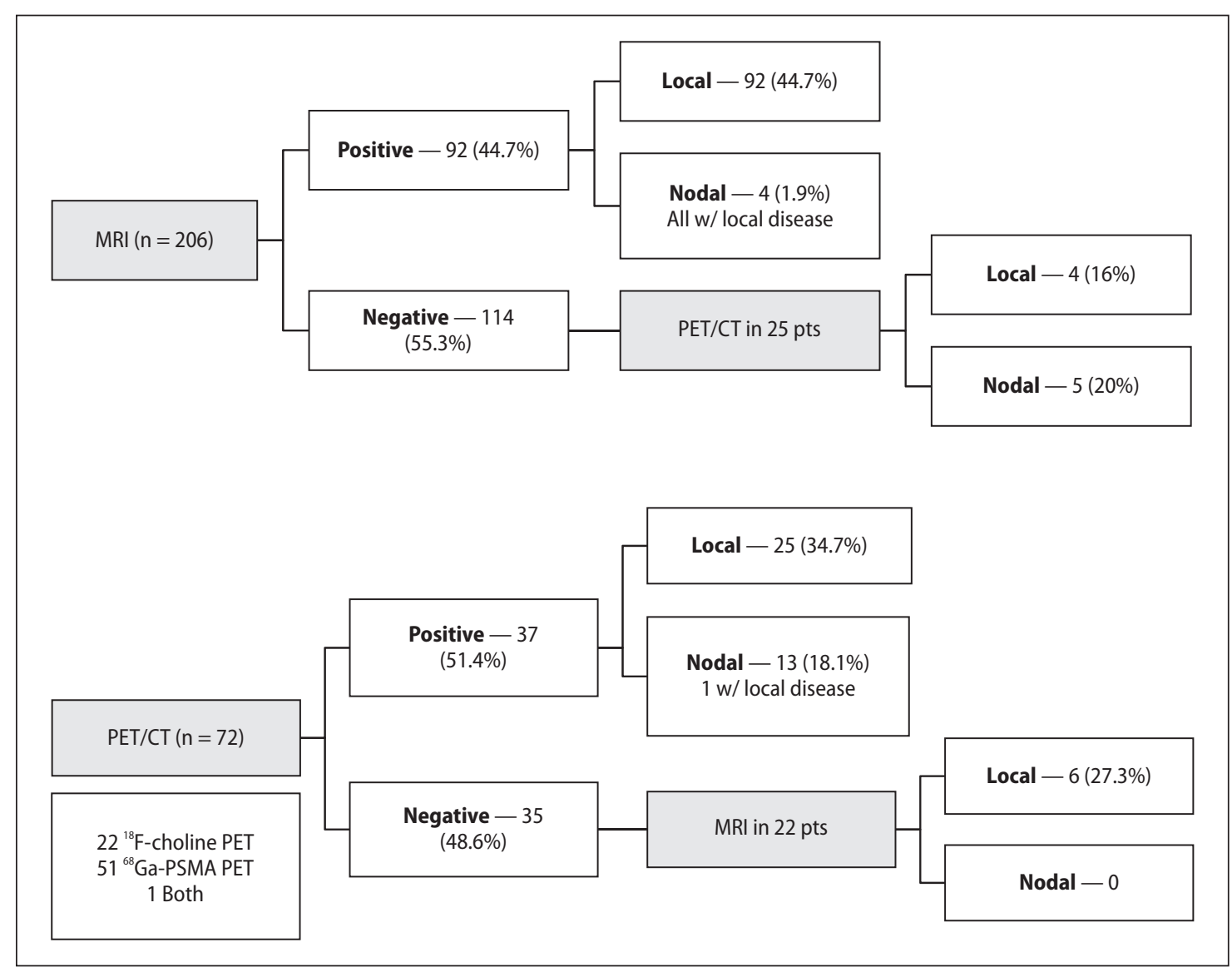

Figure 1. Restaging imaging modalities before salvage radiotherapy (sRT). MRI — magnetic resonance imaging; $\mathrm{PET} / \mathrm{CT}$ - positron emission tomography/computed tomography

tive MRI who had undergone PET/CT (whether positive or negative) with those who had not had a PET/CT done. A non-significant trend towards worse BC-RFS was observed for patients who had undergone a PET study $(\mathrm{p}=0.317)$, as well as a significantly worse C-RFS and HT-FS ( $p=0.030$ and $p=0.022$, respectively). However, this group had a significantly higher median pre-sRT PSA value (0.82 vs. $0.47 \mathrm{ng} / \mathrm{mL}, \mathrm{p}=0.027)$ and a non-significant trend towards more frequent lymphovascular invasion (36\% vs. $20 \%, \mathrm{p}=0.200)$ and negative margins (74\% vs. 64\%, $\mathrm{p}=0.377)$. Advanced $\mathrm{pT}$ stages were balanced between the two groups.

Conversely, out of 35 patients with negative PET/CT, 22 underwent MRI studies. Of these, prostate bed foci of relapse was identified in 6 (27.3\%; $8.3 \%$ of all pts who had a PET/CT), and no additional nodal disease was diagnosed. The outcome analysis also identified worse BC-RFS $(\mathrm{p}=0.043)$, C-RFS $(\mathrm{p}=0.361)$ and HT-FS ( $\mathrm{p}=0.057)$ for the PET-negative group who had undergone further imaging with MRI, whether positive or negative, in comparison with those who had not. Likewise, there was a trend towards more frequent lymphovascular invasion $(36 \% v s$. $11 \%, \mathrm{p}=0.307)$ and negative margins $(86 \% v s$. $54 \%, \mathrm{p}=0.111)$ in this group.

The positive MRI group had a significantly higher median pre-sRT in comparison with the negative MRI group (0.76 vs. $0.51 \mathrm{ng} / \mathrm{mL}, \mathrm{p}=0.001)$, with non-significant differences in the frequency of negative margins ( $71 \%$ vs. $66 \%, \mathrm{p}=0.783$ ), lymphovascular invasion $(83 \%$ vs. $77 \%, \mathrm{p}=0.435)$ and more advanced pT stages (pT3a or b in $45 \%$ vs. $36 \%$, $\mathrm{p}=0.426$ ). On the other hand, patients with a positive $\mathrm{PET} / \mathrm{CT}$ study also had higher median pre-sRT values than the negative group $(2.51 \mathrm{ng} / \mathrm{mL} v s .0 .76 \mathrm{ng} / \mathrm{mL}$, $\mathrm{p}<0.001$ ), and a non-significant trend was found towards less frequent negative margins $(74 \%$ vs. $61 \%$, $\mathrm{p}=0.244$ ), as well as more frequent lymphovascular invasion $(45 \%$ vs. $20 \%, \mathrm{p}=0.062)$ and advanced $\mathrm{pT}$ stages (pT3a or b in $50 \%$ vs. $31 \%, \mathrm{p}=0.374$ ).

Distant bone metastases were excluded by bone scintigraphy in 167 patients (60.3\%), and 28 pa- 
Table 2. Salvage radiotherapy doses and volumes

\begin{tabular}{|c|c|c|}
\hline Volume & Dose & N (\%) \\
\hline $\begin{array}{l}\text { Prostate bed } \\
277 \text { pts (100\%) }\end{array}$ & $\begin{array}{c}<66 \mathrm{~Gy} \\
66-70 \mathrm{~Gy} \\
>70 \mathrm{~Gy}\end{array}$ & $\begin{array}{c}57(20.6 \%) \\
218(78.7 \%) \\
2(0.8 \%)\end{array}$ \\
\hline $\begin{array}{l}\text { + Prostate bed boost } \\
92 \text { pts }(33.2 \%)^{*}\end{array}$ & $\begin{array}{c}68 \mathrm{~Gy} \\
70-72 \mathrm{~Gy} \\
74 \mathrm{~Gy}\end{array}$ & $\begin{array}{c}4(4.3 \%) \\
72(78.3 \%) \\
16(17.4 \%)\end{array}$ \\
\hline $\begin{array}{l}\text { Pelvic lymph nodes } \\
27 \text { pts (9.7\%) }\end{array}$ & $\begin{array}{l}45 \mathrm{~Gy} \\
50.4 \mathrm{~Gy}\end{array}$ & $\begin{array}{c}21(77.8 \%) \\
6(22.2 \%)\end{array}$ \\
\hline $\begin{array}{l}\text { + Pelvic lymph node boost } \\
11 \text { pts (40.7\%) }\end{array}$ & $\begin{array}{c}60 \mathrm{~Gy} \\
62.5-66 \mathrm{~Gy} \\
70 \mathrm{~Gy}\end{array}$ & $\begin{array}{l}5(45.5 \%) \\
2(18.2 \%) \\
4(36.4 \%)\end{array}$ \\
\hline
\end{tabular}

*Including 44 out of 57 pts who received < 66Gy to the prostate bed $(77.2 \%)$

tients (10.1\%) were referenced to our department and underwent prostate bed sRT without prior restaging imaging studies.

\section{sRT characteristics}

All patients received prostate bed irradiation, to doses $\geq 66$ Gy in $79.4 \%$ (Tab. 2). The lowest prescription dose was $60 \mathrm{~Gy}$ and the highest was $74 \mathrm{~Gy}$. A boost to prostate bed foci of disease was administered in 92 patients (33.2\%), to a total dose $\geq 72$ Gy in $54.4 \%$ (with a simultaneous integrated boost technique in 84 patients, 91.3\%). This group included most patients whose prostate bed dose was $<66 \mathrm{~Gy}$, as a way to escalate dose (44 pts, 77.2\%). Pelvic irradiation was additionally performed in 27 patients $(9.7 \%)$, most to $45 \mathrm{~Gy}(74.1 \%)$. A nodal boost was delivered in 11 patients, more frequently to total doses of $60 \mathrm{~Gy}$ (45.5\%) or 70Gy (36.4\%). Most were planned with an IMRT technique (86.3\%), and the remaining with VMAT. Androgen deprivation therapy (ADT) was prescribed for 18 patients (6.5\%), a median of 29.4 months (range: $1.4-83.5$ ).

\section{Treatment outcomes, patterns of failure}

Outcome analysis was performed for 264 patients, for whom follow-up information was available. Median follow-up was 35.6 months (4.5-86.8). At 3 years, BC-RFS was $61.4 \%$, C-RFS was $81.3 \%$, HT-FS was $79.9 \%$ and OS was $96.6 \%$. At the end of follow-up, a biochemical failure had been diagnosed in 101 patients (38.3\%), and 52 patients had had a clinical relapse (19.7\%). Out of 48 patients who suffered clinical relapse during follow-up (18.2\%), the most common location was the regional lymph nodes alone $(\mathrm{n}=23 ; 47.9 \%)$, followed by distant relapse $(n=12 ; 25.0 \%)$, regional nodal and distant relapse $(n=8 ; 16.7 \%)$, prostate bed alone $(\mathrm{n}=3 ; 6.2 \%)$, prostate bed and regional lymph nodes $(\mathrm{n}=1 ; 2.1 \%)$ and prostate bed, nodal and distant relapse $(\mathrm{n}=1 ; 2.1 \%)$. Four of the 23 patients who suffered regional nodal-only relapse had not been imagiologically evaluated prior to sRT (17.4\%, whose clinical relapse was diagnosed a median of 40 months post-sRT). Surgical margins had been negative in most of these patients ( $\mathrm{n}=18 ; 81.8 \%)$.

Thirty-four patients showed PSA persistence on the first measurement after sRT (12.9\%) - their median pre-sRT PSA was non-significantly higher than that of patients without PSA persistence (0.75 vs. $0.58 \mathrm{ng} / \mathrm{mL}, \mathrm{p}=0.193$ ).

In opposition, undetectable PSA was achieved in 75 patients $(28.4 \%)$, a median of 11.2 months after sRT (from 2 to 52 months). Median pre-sRT PSA was significantly lower in this population, compared with the group who did not achieve undetectable PSA $(0.42$ vs. $0.65 \mathrm{ng} / \mathrm{mL}, \mathrm{p}<0.01)$. In the undetectable PSA group, only 5 patients later suffered a biochemical relapse (6.7\%) and 2 had a clinical relapse $(2.7 \%)$. Among the group that did not achieve undetectable PSA, biochemical relapse was significantly more common $(48.1 \%, \mathrm{p}<0.01)$, as well as clinical relapse $(24.3 \%, \mathrm{p}<0.01)$.

\section{Impact of disease characteristics on the outcome}

On MVA retaining relevant variables previously studied in UVA (Tab. 3), we observed that BC-RFS was negatively influenced by lymphovascular invasion $(\mathrm{p}=0.029)$, advanced $\mathrm{pT}$ stages $(\mathrm{p}=0.002)$ and negative surgical margin after RP $(\mathrm{p}<0.001)$. HT-FS was also negatively impacted by these 3 factors $(\mathrm{p}=0.001, \mathrm{p}=0.029$ and $\mathrm{p}=0.002$, respectively). The presence of lymphovascular invasion and negative surgical margins also worsened C-RFS ( $p=0.009$ and $p=0.015$, respectively), but OS was not affected by any of these.

\section{Impact of sRT timing}

sRT was started with PSA $>0.5 \mathrm{ng} / \mathrm{mL}$ in 144 patients who had follow-up data (54.4\% - group B). Group B had a higher prevalence of pT3a or b tumors (51.8 vs. $32.7 \%, \mathrm{p}=0.001$ ), but the remaining characteristics that had been tested were balanced 
Table 3. Multivariate analysis for each outcome

\begin{tabular}{|c|c|c|c|}
\hline Outcome & Variable & p-value & HR $(95 \% \mathrm{Cl})$ \\
\hline \multirow{9}{*}{ BC-RFS } & Lymphovascular invasion & 0.029 & $1.956(1.071-3.573)$ \\
\hline & pT Stage (vs. pT2a) & 0.002 & \\
\hline & pT2b & 0.463 & $2.861(0.173-47.380)$ \\
\hline & pT2c & 0.084 & $5.909(0.789-44.271)$ \\
\hline & рT3a & 0.014 & $12.480(1.675-92.981)$ \\
\hline & pT3b & 0.003 & 24.267 (3.034-194.105) \\
\hline & Surgical margin (vs. negative) & $<0.001$ & \\
\hline & Positive & 0.003 & $0.379(0.200-0.717)$ \\
\hline & Tangential & 0.002 & $0.231(0.091-0.589)$ \\
\hline \multirow{9}{*}{ HT-FS } & Lymphovascular invasion & 0.001 & 3.697 (1.726-7.918) \\
\hline & pT Stage (vs. pT2a) & 0.029 & \\
\hline & $\mathrm{pT} 2 \mathrm{~b}$ & 0.980 & $0.000(0.000)$ \\
\hline & pT2c & 0.270 & $3.164(0.408-24.535)$ \\
\hline & рT3a & 0.237 & $3.454(0.443-26.933)$ \\
\hline & pT3b & 0.024 & $11.247(1.375-92.028)$ \\
\hline & Surgical margin (vs. negative) & 0.002 & \\
\hline & Positive & 0.009 & $0.318(0.135-0.749)$ \\
\hline & Tangential & 0.011 & $0.071(0.009-0.539)$ \\
\hline \multirow{4}{*}{ C-RFS } & Lymphovascular invasion & 0.009 & $2.698(1.287-5.655)$ \\
\hline & Surgical margin (vs. negative) & 0.006 & \\
\hline & Positive & 0.016 & $0.368(0.163-0.831)$ \\
\hline & Tangential & 0.015 & $0.164(0.038-0.704)$ \\
\hline
\end{tabular}

BC-RFS — biochemical relapse-free survival; HT-FS — additional hormone therapy-free survival; C-RFS — clinical relapse-free survival

between the two (grade group, surgical margins, perineural invasion, lymphovascular invasion, initial PSA value, post-RP undetectable PSA, adjuvant ADT). Median time from relapse to start of sRT was also longer in group B than group A (13.1 vs. 5.5 months, $\mathrm{p}<0.001$ ).

BC-RFS was significantly superior on group A (67.8 vs. $56.0 \%$ at 3 years, $\mathrm{p}=0.011)$, as well as HT-FS (86.6 vs. $74.6 \%$ at 3 years, $\mathrm{p}=0.001)$. No significant differences were found on C-RFS $(82.3$ vs. $80.6 \%$ at 3 years, $\mathrm{p}=0.324)$ or OS ( $96.8 v$ s. $96.5 \%$ at 3 years, $\mathrm{p}=0.479$ ) (Fig. 2).

\section{Discussion}

In our study, we sought to determine the most significant factors influencing outcomes after sRT. Upon biochemical relapse after RP, the use of either MRI or PET/CT for restaging is recommended by the EUA guidelines in the case when findings alter treatment decisions. [9] MRI is recommended by ESUR for pelvic evaluation in patients with low PSA levels $(0.2-2 \mathrm{ng} / \mathrm{mL})$, with detection rates that vary from $24 \%$ to $91 \%$ [10] Conversely, on a sur- vey by Panje et al. all the centers recommended ${ }^{18} \mathrm{~F}$-choline or ${ }^{68} \mathrm{Ga}$-PSMA PET/CT for restaging, and it has been reported to change management in $55 \%$ patients $[11,12]$. According to a meta-analysis, although with a suboptimal sensitivity, ${ }^{18} \mathrm{~F}$-choline $\mathrm{PET} / \mathrm{CT}$ is more accurate for lymph node detection than MRI [13]. In addition, ${ }^{68} \mathrm{Ga}$-PSMA PET/CT shows excellent specificity and positive predictive values for lymph node detection, with per-patient sensitivity ranging from 33.3 to $100 \%$ [14]. In order to improve the detection of pelvic relapses, some authors have proposed combining the two techniques [10]. Although there may be a bias, we found that these techniques complemented each other in a relevant percentage of cases (with PET/CT identifying additional foci of disease in $36 \%$ of patients with negative MRI who underwent PET/CT, and MRI diagnosing prostate bed relapse in $27.3 \%$ of patients with negative PET/CT who underwent MRI). There is relevant heterogeneity in studies evaluating this topic, but some series have suggested that these imaging studies have a potential impact on outcomes (with better treatment response in PET-negative patients or in those with prostate bed foci [15]; and 


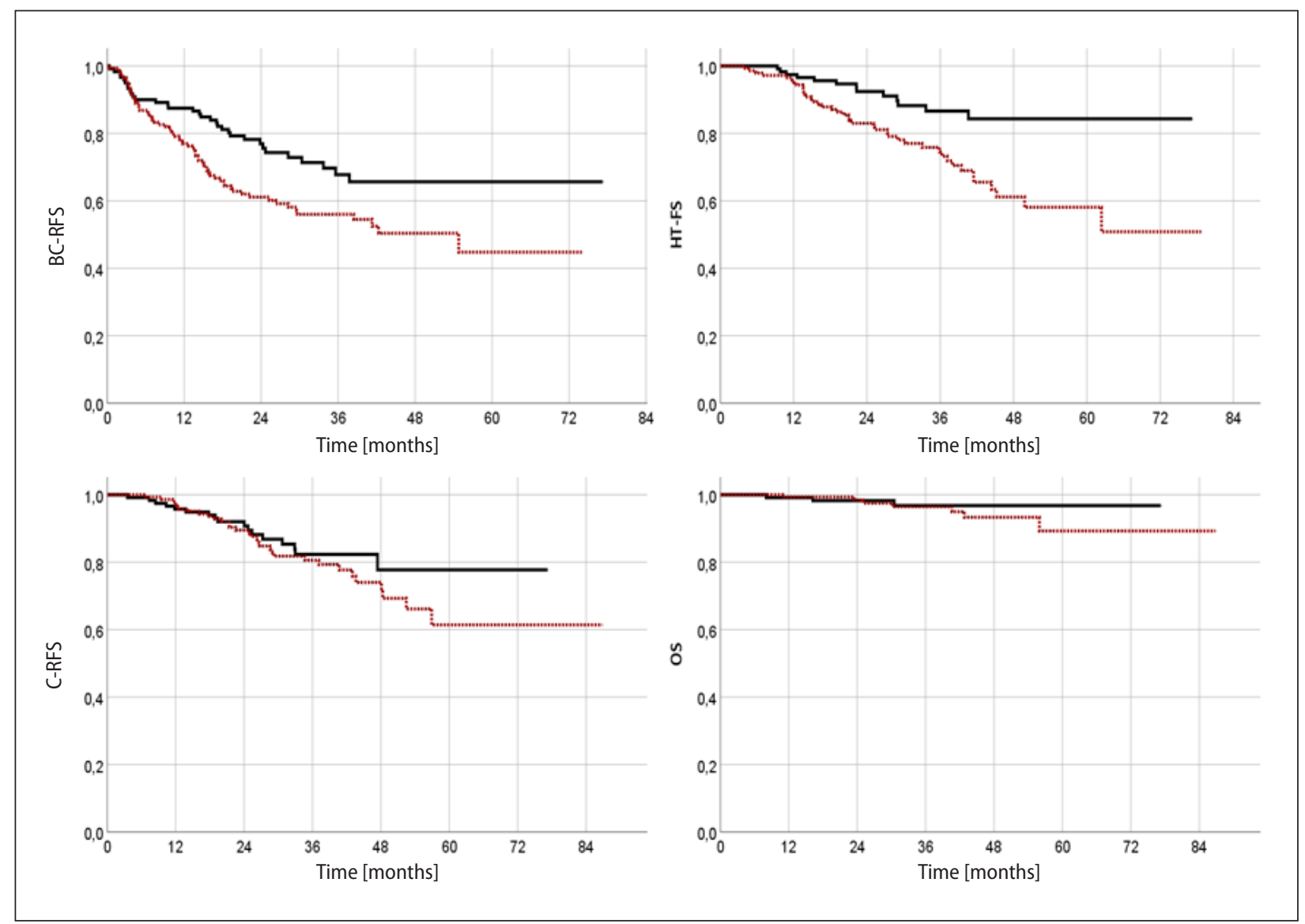

Figure 2. Survival analysis comparison between Group A (pre-sRT PSA $\leq 0.5 \mathrm{ng} / \mathrm{mL}$, black solid line) and Group B (pre-sRT PSA > $0.5 \mathrm{ng} / \mathrm{mL}$, red dotted line); SRT — salvage radiotherapy; PSA — prostate-specific antigen

an improvement in prognostication when MRI is analysed together with other clinical and pathological variables after sRT [16]) and treatment decisions (with $63 \%$ nodal relapses identified by ${ }^{68} \mathrm{Ga}$-PSMA PET/CT being located on the pelvis and lumboaortic region and $95 \%$ of them being amenable to sRT [17-19]; and the possibility of MRI-guided adaptive radiotherapy or boosts, reported by several authors [10]). Although we found worse outcomes for patients with negative MRI who had undergone additional PET/CT, the higher pre-sRT PSA value and trend towards more frequent negative prognosis factors in this group suggests these differences may be explained by an attempt to pursue further imaging studies in perceived worse prognosis patients, creating a selection bias. The same was observed for the negative-PET/CT group who underwent MRI [21].

In our cohort, few patients received salvage ADT. In fact, results from RTOG 9601 (24 months bicalutamide during and after sRT) [22] and GETUG-AFU 16 (goserelin on the first day of sRT and at 3 months) [23] have since supported the use of concomitant ADT, which is now recommended by ASTRO/AUA guidelines. [24] Since this cohort was treated before long follow-up results from these randomized clinical trials were made available, ADT was not standard in our Institution and, therefore, PSA follow-up was included from the conclusion of sRT in most patients.

We observed a 3-year BC-RFS of $61.4 \%$, which is comparable to that reported in a systematic review, of 57-64\% [25]. Overall, clinical recurrence was noted in $18.2 \%$ patients, predominantly in the regional lymph nodes alone (in $47.9 \%$ clinically relapsed patients). Of these, $17.4 \%$ had not been radiologically staged by MRI or PET/CT prior to sRT. In spite of a relatively long median time to clinical relapse (40 months), this emphasizes the importance of adequate systematic imaging studies prior to sRT. Our predominant pattern of failure and its crude rate (23 in 264 patients, $8.9 \%$ ) are in agreement with data from Brand et al. (who report 
crude nodal-only relapse rates of $11 \%$ with a 7 year follow-up), especially given that our follow-up time is shorter [26].

Patients with positive margins after RP have been reported to suffer biochemical recurrence and undergo RT more frequently [27, 28]. In these cases, it is more likely that the focus of relapse is on the prostate bed. Since all of our patients received sRT to a prostate bed volume, it is then probable that they received sRT to the site of relapse, whereas foci of disease on negative margin patients could be occult on imaging studies and, therefore, not targeted. Otherwise, the residual disease could have driven biochemical relapse for positive margin patients, whereas the negative margin population could correspond to more intrinsically aggressive tumors. These are hypothesis that could explain the observed "protective" influence of a positive margin on outcomes after sRT in this population. This finding was also observed in several retrospective studies, such as those by Jackson, Ervandian, Galla, Fossati and Goenka who noted better outcomes in patients with positive margins after sRT [29, 30, 31, 32].

According to several retrospective studies, a higher pathological $\mathrm{T}$ stage is associated with poorer biochemical relapse-free survival, which our data corroborates. Some have also correlated higher stages with lower metastasis-free survival and prostate cancer-specific survival [29, 31].

Lymphovascular invasion has also been linked with poor prognosis after sRT. Worse biochemical failure-free survival after sRT has been reported in several retrospective series. Jeong et al. also described significantly worse clinical failure-free survival and cancer-specific survival for patients with lymphovascular invasion [33].

We observed more favourable BC-RFS and HT-FS for patients who had undergone early sRT (which was defined as sRT with a PSA $\leq 0.5$ $\mathrm{ng} / \mathrm{mL}$ ). No significant differences were observed on C-RFS and OS, possibly due to a low number of events (although a trend for more favourable C-RFS and OS was observed for Group A). The definition of "early sRT" is widely variable, including the one used in ongoing randomized trials comparing adjuvant radiotherapy with early sRT: on RADICALS-RT, treatment is initiated when biochemical failure is diagnosed (defined by three consecutive rises in PSA, or two consecutive rises with a final PSA $>0.1 \mathrm{ng} / \mathrm{mL}$ ) [3]; on RAVES, sRT is triggered by PSA $\geq 0.2 \mathrm{ng} / \mathrm{mL}$ [4]. Nevertheless, the benefit of early rather than late sRT has been broadly described in the literature, with Stish et al. reporting a significant increase in biochemical recurrence, distant metastasis, cancer specific mortality and all-cause mortality with each pre-sRT PSA doubling. Additionally, they reported a significantly lower incidence of biochemical relapse, distant metastasis and cancer specific mortality when pre-sRT PSA was $\leq 0.5 \mathrm{ng} / \mathrm{mL}[34]$.

Driven by its retrospective nature, our study has several limitations, including possible missing data. The fact that patients later underwent different surveillance protocols (according to their attending physician) may also limit data interpretation, since the periodicity of PSA dosing, clinical evaluation and the threshold for requesting additional imaging studies may have varied. In addition, criteria for requesting imaging studies to characterize initial relapse before sRT varied, which may be responsible for some bias regarding the analysis of how MRI and PET/CT performed in such setting. The median follow-up time (35.6 months) is also short for prostate cancer.

\section{Conclusion}

We highlighted the importance of adequate restaging prior to sRT, with occult disease in MRI having been identified on PET/CT, and vice-versa. The primary pattern of relapse in our cohort was in the regional lymph nodes, with similar outcomes to those described in the literature. Prognosis was negatively influenced by the presence of lymphovascular invasion, higher pathological T-stages and negative margins, but an early start of sRT (namely with PSA $\leq 0.5$ $\mathrm{ng} / \mathrm{mL}$ ) was a predictor of better BC-RFS and HT-FS. Therefore, a prompt diagnosis of relapse and restaging, especially in patients with adverse prognostic factors, may help improve prognosis.

\section{Conflicts of interest}

None to declare.

\section{Funding}

This research did not receive any grants or funding.

\section{Ethics committee}

Approval was obtained from our Institutional Ethics Committee (CES - 227/020). 


\section{References}

1. Fersino S, Tebano U, Mazzola R, et al. Moderate Hypofractionated Postprostatectomy Volumetric Modulated Arc Therapy With Daily Image Guidance (VMAT-IGRT): A Mono-institutional Report on Feasibility and Acute Toxicity. Clin Genitourin Cancer. 2017; 15(4): e667e673, doi: 10.1016/j.clgc.2017.01.025, indexed in Pubmed: 28237181.

2. Cozzarini C, Fiorino C, Briganti A, et al. Higher-thanexpected severe (Grade 3-4) late urinary toxicity after postprostatectomy hypofractionated radiotherapy: a single-institution analysis of 1176 patients. Eur Urol. 2014;66(6): 1024-1030, doi: 10.1016/j.eururo.2014.06.012, indexed in Pubmed: 24985964.

3. Parker CC, Clarke NW, Cook AD, et al. Timing of radiotherapy after radical prostatectomy (RADICALSRT): a randomised, controlled phase 3 trial. Lancet. 2020; 396(10260): 1413-1421, doi: 10.1016/S01406736(20)31553-1, indexed in Pubmed: 33002429.

4. Kneebone A, Fraser-Browne C, Delprado W, et al. A Phase III Multi-Centre Randomised Trial comparing adjuvant versus early salvage Radiotherapy following a Radical Prostatectomy: Results of the TROG 08.03 and ANZUP "RAVES" Trial. Int J Radiat Oncol Biol Phys. 2019; 105(1): S37-S38, doi: 10.1016/j.ijrobp.2019.06.456.

5. Sargos P, Chabaud S, Latorzeff I, et al. Adjuvant radiotherapy versus early salvage radiotherapy plus short-term androgen deprivation therapy in men with localised prostate cancer after radical prostatectomy (GETUGAFU 17): a randomised, phase 3 trial. Lancet Oncol. 2020; 21(10): 1341-1352, doi: 10.1016/S1470-2045(20)30454-X, indexed in Pubmed: 33002438.

6. Tourinho-Barbosa R, Srougi V, Nunes-Silva I, et al. Biochemical recurrence after radical prostatectomy: what does it mean? Int Braz J Urol. 2018; 44(1): 14-21, doi: 10.1590/S1677-5538.IBJU.2016.0656, indexed in Pubmed: 29039897.

7. Jackson WC, Suresh K, Tumati V, et al. Impact of Biochemical Failure After Salvage Radiation Therapy on Prostate Cancer-specific Mortality: Competition Between Age and Time to Biochemical Failure. Eur Urol Oncol. 2018; 1(4): 276-282, doi: 10.1016/j.euo.2018.04.014, indexed in Pubmed: 31100248.

8. Do T, Parker RG, Do C, et al. Salvage radiotherapy for biochemical and clinical failures following radical prostatectomy. Cancer J Sci Am. 1998; 4(5): 324-330, indexed in Pubmed: 9815297.

9. Mottet N, Cornford P, van den Bergh RCN et al. EAU Prostate Cancer Guidelines. https://uroweb.org/guideline/ prostate-cancer/ (9 June 2020).

10. Couñago F, Sancho G, Catalá V, et al. Magnetic resonance imaging for prostate cancer before radical and salvage radiotherapy: What radiation oncologists need to know. World J Clin Oncol. 2017; 8(4): 305-319, doi: 10.5306/wjco. v8.i4.305, indexed in Pubmed: 28848697.

11. Panje C, Zilli T, Dal Pra A, et al. Radiotherapy for pelvic nodal recurrences after radical prostatectomy: patient selection in clinical practice. Radiat Oncol. 2019; 14(1): 177, doi: 10.1186/s13014-019-1383-0, indexed in Pubmed: 31619296.
12. Goldstein J, Even-Sapir E, Ben-Haim S, et al. Does Choline PET/CT Change the Management of Prostate Cancer Patients With Biochemical Failure? Am J Clin Oncol. 2017; 40(3): 256-259, doi: 10.1097/COC.0000000000000139, indexed in Pubmed: 25319322.

13. Huang SM, Yin L, Yue JL, et al. Direct comparison of choline PET/CT and MRI in the diagnosis of lymph node metastases in patients with prostate cancer. Medicine (Baltimore). 2018; 97(50): e13344, doi: 10.1097/ MD.0000000000013344, indexed in Pubmed: 30557983.

14. Luiting HB, van Leeuwen PJ, Busstra MB, et al. Use of gallium-68 prostate-specific membrane antigen positronemission tomography for detecting lymph node metastases in primary and recurrent prostate cancer and location of recurrence after radical prostatectomy: an overview of the current literature. BJU Int. 2020; 125(2): 206-214, doi: 10.1111/bju.14944, indexed in Pubmed: 31680398.

15. Emmett L, van Leeuwen PJ, Nandurkar R, et al. Treatment Outcomes from Ga-PSMA PET/CT-Informed Salvage Radiation Treatment in Men with Rising PSA After Radical Prostatectomy: Prognostic Value of a Negative PSMA PET. J Nucl Med. 2017; 58(12): 1972-1976, doi: 10.2967/ jnumed.117.196683, indexed in Pubmed: 28747524.

16. Sharma V, Nehra A, Colicchia M, et al. Multiparametric Magnetic Resonance Imaging Is an Independent Predictor of Salvage Radiotherapy Outcomes After Radical Prostatectomy. Eur Urol. 2018; 73(6): 879-887, doi: 10.1016/j. eururo.2017.11.012, indexed in Pubmed: 29195777.

17. De Bari B, Mazzola R, Aiello D, et al. (Ga)-PSMA-PET/ $\mathrm{CT}$ for the detection of postoperative prostate cancer recurrence: Possible implications on treatment volumes for radiation therapy. Cancer Radiother. 2019; 23(3): 194-200, doi: 10.1016/j.canrad.2018.09.003, indexed in Pubmed: 31088725.

18. De Bari B, Mazzola R, Aiello D, et al. Could 68-Ga PSMA $\mathrm{PET} / \mathrm{CT}$ become a new tool in the decision-making strategy of prostate cancer patients with biochemical recurrence of PSA after radical prostatectomy? A preliminary, monocentric series. Radiol Med. 2018; 123(9): 719-725, doi: 10.1007/s11547-018-0890-7, indexed in Pubmed: 29687208.

19. Alongi F, Fersino S, Giaj Levra N, et al. Impact of $18 \mathrm{~F}$ Choline PET/CT in the Decision-Making Strategy of Treatment Volumes in Definitive Prostate Cancer Volumetric Modulated Radiation Therapy. Clin Nucl Med. 2015; 40(11): e496-e500, doi: 10.1097/RLU.0000000000000841, indexed in Pubmed: 26053712.

20. Couñago F, Díaz Gavela AA, Sancho G, et al. Multiparametric magnetic resonance imaging-guided salvage radiotherapy in prostate cancer. Rep Pract Oncol Radiother. 2019; 24(5): 472-480, doi: 10.1016/j.rpor.2019.07.008, indexed in Pubmed: 31452628.

21. Mazzola R, Francolini G, Triggiani L, et al. Metastasisdirected Therapy (SBRT) Guided by PET-CT F-CHOLINE Versus PET-CT Ga-PSMA in Castration-sensitive Oligorecurrent Prostate Cancer: A Comparative Analysis of Effectiveness. Clin Genitourin Cancer. 2020 [Epub ahead of print], doi: 10.1016/j.clgc.2020.08.002, indexed in Pubmed: 32863189.

22. Baumgarten L, Borchert A, Sood A, et al. Impact of timing on salvage radiation therapy adverse events follow- 
ing radical prostatectomy: A secondary analysis of the RTOG 9601 cohort. Urol Oncol. 2020; 38(2): 38.e17-38. e22, doi: 10.1016/j.urolonc.2019.09.012, indexed in Pubmed: 31653564.

23. Carrie $C$, Magné N, Burban-Provost $P$, et al. Short-term androgen deprivation therapy combined with radiotherapy as salvage treatment after radical prostatectomy for prostate cancer (GETUG-AFU 16): a 112-month followup of a phase 3, randomised trial. Lancet Oncol. 2019; 20(12): 1740-1749, doi: 10.1016/S1470-2045(19)30486-3, indexed in Pubmed: 31629656.

24. Pisansky TM, Thompson IM, Valicenti RK, et al. Adjuvant and Salvage Radiotherapy after Prostatectomy: ASTRO/ AUA Guideline Amendment 2018-2019. J Urol. 2019; 202(3): 533-538, doi: 10.1097/JU.0000000000000295, indexed in Pubmed: 31042111.

25. Bianchi L, Gandaglia G, Fossati N, et al. Adjuvant Radiotherapy in Prostate Cancer Patients Treated with Surgery: The Impact of Age and Tumor Characteristics. Eur Urol Focus. 2015; 1(2): 191-199, doi: 10.1016/j.euf.2015.06.008, indexed in Pubmed: 28723433.

26. Brand DH, Parker JI, Dearnaley DP, et al. Patterns of recurrence after prostate bed radiotherapy. Radiother Oncol. 2019; 141: 174-180, doi: 10.1016/j.radonc.2019.09.007, indexed in Pubmed: 31563410.

27. Zhang L, Wu B, Zha Z, et al. Surgical margin status and its impact on prostate cancer prognosis after radical prostatectomy: a meta-analysis. World J Urol. 2018; 36(11): 1803-1815, doi: 10.1007/s00345-018-2333-4, indexed in Pubmed: 29766319.

28. Kvåle R, Myklebust TÅ, Fosså SD, et al. Impact of positive surgical margins on secondary treatment, palliative radiotherapy and prostate cancer-specific mortality. A population-based study of 13198 patients. Prostate.
2019; 79(16): 1852-1860, doi: 10.1002/pros.23911, indexed in Pubmed: 31566779.

29. Jackson W, Hamstra DA, Johnson S, et al. Gleason pattern 5 is the strongest pathologic predictor of recurrence, metastasis, and prostate cancer-specific death in patients receiving salvage radiation therapy following radical prostatectomy. Cancer. 2013; 119(18): 3287-3294, doi: 10.1002/cncr.28215, indexed in Pubmed: 23821578.

30. Ervandian M, Høyer M, Petersen SE, et al. Salvage radiation therapy following radical prostatectomy. A national Danish study. Acta Oncol. 2016; 55(5): 598-603, doi: 10.3109/ 0284186X.2015.1088170, indexed in Pubmed: 26399602.

31. Fossati N, Karnes RJ, Cozzarini C, et al. Assessing the Optimal Timing for Early Salvage Radiation Therapy in Patients with Prostate-specific Antigen Rise After Radical Prostatectomy. Eur Urol. 2016; 69(4): 728-733, doi: 10.1016/j. eururo.2015.10.009, indexed in Pubmed: 26497924.

32. Goenka A, Magsanoc JM, Pei X, et al. Long-term outcomes after high-dose postprostatectomy salvage radiation treatment. Int J Radiat Oncol Biol Phys. 2012; 84(1): 112-118, doi: 10.1016/j.ijrobp.2011.10.077, indexed in Pubmed: 22300563.

33. Jeong JU, Nam TK, Song JY, et al. Prognostic significance of lymphovascular invasion in patients with prostate cancer treated with postoperative radiotherapy. Radiat Oncol J.2019; 37(3):215-223, doi: 10.3857/roj.2019.00332, indexed in Pubmed: 31591870.

34. Stish BJ, Pisansky TM, Harmsen WS, et al. Improved Metastasis-Free and Survival Outcomes With Early Salvage Radiotherapy in Men With Detectable Prostate-Specific Antigen After Prostatectomy for Prostate Cancer. J Clin Oncol. 2016; 34(32): 3864-3871, doi: 10.1200/JCO.2016.68.3425, indexed in Pubmed: 27480153. 